\title{
RAMANUJAN GRAPHS AND HECKE OPERATORS
}

\author{
ARNOLD K. PIZER
}

\section{INTRODUCTION}

We associate to the Hecke operator $T_{p}, p$ a prime, acting on a space of theta series an explicit $p+1$ regular Ramanujan graph $G$ having large girth. Such graphs have high "magnification" and thus have many applications in the construction of networks and explicit algorithms (see [LPS1] and Bien's survey article [B]). In general our graphs do not seem to have quite as large a girth as the Ramanujan graphs discovered by Lubotzky, Phillips, and Sarnak ([LPS1, LPS3]) and independently by Margulis ([M]). However, by varying the $T_{p}$ and the spaces of theta series, we obtain a much larger family of interesting graphs. The trace formula for the action of the Hecke operators $T_{p^{r}}$ immediately yields information on certain closed walks in $G$ and in particular on the girth of $G$. If $m$ is not a prime, we obtain "almost Ramanujan" graphs associated to $T_{m}$.

The results of this paper can be viewed as an explicit version of a generalization of a construction of Ihara (see [I] and Theorem 4.1 of [LPS2]). From this viewpoint the connection between our results and those of Lubotzky, Phillips, and Sarnak becomes clearer. Recently, Chung ([C]) and Li ([L]) also constructed Ramanujan graphs associated to certain abelian groups.

\section{GRAPHS}

Let $G$ be a multigraph (i.e., we allow loops and multiple edges) with $n$ vertices $v_{i}$ and edges $e_{j}$. A walk $W$ on $G$ is an alternating sequence of vertices and edges $v_{0} e_{1} v_{1} e_{2} v_{2} \ldots e_{r} v_{r}$ where each edge $e_{j}$ has endpoints $v_{j-1}$ and $v_{j}$. We say $W$ is a walk from $v_{0}$ to $v_{r}$ of length $r$. $W$ is closed if and only if $v_{r}=v_{0}$. A walk is said to be without backtracking (a w.b. walk) if a "point can transverse the walk without stopping and backtracking." The only

Received by the editors May 25, 1989 and, in revised form, December 5, 1989.

1980 Mathematics Subject Classification (1985 Revision). Primary 05C35; Secondary $11 \mathrm{~F} 25$. 
subtleness in this vague definition occurs when $G$ contains loops. Rather than giving a precise definition here (for this see [S]), we illustrate the definition with Examples 1 and 2 which make the idea clear.

Example 1. Let $G$ be the multigraph:

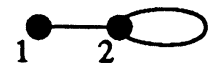

Then $G$ has respectively 0,1 , and 2 w.b. walks of length 1 from $v_{1}$ to $v_{1}$, from $v_{1}$ to $v_{2}$ (or $v_{2}$ to $v_{1}$ ), and from $v_{2}$ to $v_{2}$. G has respectively 0,2 , and 2 w.b. walks of length 2 from $v_{1}$ to $v_{1}$, from $v_{1}$ to $v_{2}$ (or $v_{2}$ to $v_{1}$ ), and from $v_{2}$ to $v_{2}$. For all $r \geq 3, G$ has exactly 2 w.b. walks of length $r$ from $v_{i}$ to $v_{j}$, $1 \leq i, j \leq 2$.

Let $a_{i j}^{(r)}$ denote the number of w.b. walks of length $r$ from $v_{i}$ to $v_{j}$ in $G$ and put $A_{r}=\left(a_{i j}^{(r)}\right)$. The $A_{r}$ are symmetric $n$ by $n$ matrices with nonnegative integer entries and even diagonal entries. $A_{1}$ is the adjacency matrix of $G . G$ is determined by $A_{1}$ and every symmetric $n$ by $n$ matrix with nonnegative integer entries and even diagonal entries determines a multigraph. It is clear that $G$ has no loops if and only if $\operatorname{tr}\left(A_{1}\right)=0$ and that $G$ is a graph (i.e., $G$ has neither loops nor multiple edges) if and only if $\operatorname{tr}\left(A_{2}\right)=0$. Further the girth of $G$ is the smallest positive integer $g$ such that $\operatorname{tr}\left(A_{g}\right)>0$ and if $\operatorname{tr}\left(A_{r}\right)=0$, then $G$ has no cycles of length $r$. The least $d$, if it exists, for which for every pair $i, j, 1 \leq i<j \leq n$, there exists an $r=r(i, j) \leq d$ with $a_{i j}^{(r)}>0$, is called the diameter of $G$. A (finite) multigraph with a finite diameter is said to be connected. Let $d_{i i}=\sum_{j=1}^{n} a_{i j}^{(1)}$ be the degree of $v_{i}$ and let $D$ be the diagonal matrix with diagonal entries $d_{i i}$. Let $I$ be the $n$ by $n$ identity matrix. The $A_{r}$ are determined recursively by

Proposition 1. Let the notation be as above. Then

$$
\begin{aligned}
& A_{1} A_{1}=A_{2}+D \\
& A_{r} A_{1}=A_{r+1}+A_{r-1}(D-I) \quad \text { for } r \geq 2 .
\end{aligned}
$$

Example 2. Let $G$ be the multigraph:

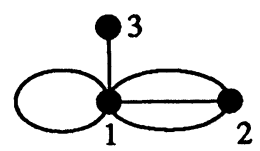


Then $D$ has diagonal entries 6,3 , and 1 and

$$
A_{1}=\left(\begin{array}{lll}
2 & 3 & 1 \\
3 & 0 & 0 \\
1 & 0 & 0
\end{array}\right) \quad A_{2}=\left(\begin{array}{lll}
8 & 6 & 2 \\
6 & 6 & 3 \\
2 & 3 & 0
\end{array}\right) \quad A_{3}=\left(\begin{array}{ccc}
26 & 18 & 8 \\
18 & 18 & 6 \\
8 & 6 & 2
\end{array}\right)
$$

We define $n$ by $n$ matrices $B_{r}$ recursively as follows:

$$
\begin{aligned}
& B_{-1}=0, \quad B_{0}=I, \quad B_{1}=A_{1}, \quad \text { and } \\
& B_{r} B_{1}=B_{r+1}+B_{r-1}(D-I)
\end{aligned}
$$

for $r \geq 0$. The relation between the $A_{r}$ and $B_{r}$ is given by

Proposition 2. $A_{r}=B_{r}-B_{r-2}$ for all $r \geq 1$.

Remark 1. Assume $G$ is a $k$ regular multigraph (i.e., the degree of each vertex is $k$ ) with $k=p+1$ for some prime $p$. Then (2) becomes

$$
B_{r} B_{1}=B_{r+1}+p B_{r-1} \quad \text { for } \quad r \geq 0 .
$$

This is exactly the recursion relation satisfied by the Hecke operators $B_{r}=T_{p^{r}}$ acting on a space of modular forms of weight 2 on $\Gamma_{0}(N)$ when $p \nmid N$. Thus if we are able to associate a $p+1$ regular graph $G$ to the Hecke operator $T_{p}$, the action of the Hecke operators $T_{p^{r}}$ will, by Proposition 2, determine the $A_{r}$ and hence give us information about $G$. For example a trace formula for $T_{p^{r}}$ immediately yields information on the girth of $G$. This is precisely what we intend to do in this announcement. By varying the spaces on which the Hecke operators act, we will obtain a large family of interesting (e.g., Ramanujan with relatively large girth) graphs. If $m$ is not prime, we will also be able to associate a graph to $T_{m}$. These graphs will in general be "almost Ramanujan."

\section{Quaternion Algebras}

For simplicity in this announcement we consider only quaternion algebras $\mathbb{A}$ over $\mathbb{Q}$ ramified precisely at one finite prime $q \geq 5$ and $\infty$ (but see Remark 2 below). See [P4] Proposition 5.1 for an explicit description of these $\mathbb{A}$. Let $M$ be a positive integer prime to $q$ and let $\mathscr{O}$ be an order of level $N=q^{2} M$ contained in $\mathbb{A}$ (see Definition 3.5 and Theorem 1.5 of [P3] and Remark 2 below). If $m$ is a positive integer relatively prime to $N$, the Hecke operator $T_{m}$ acts on a space of theta series (which are modular forms of weight 2 on $\left.\Gamma_{0}(N)\right)$ associated to $\mathscr{O}$ and this action has an explicit matrix representation given by the Brandt 
matrix $B(m)=B\left(q^{2}, M ; m\right)$ ([E, HS, P3, P4, HPS2]). The Brandt matrix is described in terms of the arithmetic of $\mathscr{O}$ as follows. Let $I_{1}, \ldots, I_{H}$ be representatives of all the distinct left $\mathscr{O}$-ideal classes. Here $H$, the class number of $\mathscr{O}$, is given by the formula (Theorem 4.8 of [P3])

$$
H=\left(\frac{q^{2}-1}{12}\right) M \prod_{\ell \mid M}(1+1 / \ell),
$$

where the product is over all the distinct primes $\ell$ dividing $M$. Let $b_{i j}(m)$ denote $e_{j}^{-1}$ times the number of $\alpha$ in $I_{j}^{-1} I_{i}$ with $\mathrm{N}(\alpha)=m \mathrm{~N}\left(I_{i}\right) / \mathrm{N}\left(I_{j}\right)$. Here $e_{j}$ is the number of units in the right order of $I_{j}$ and $\mathrm{N}()$ denotes the reduced norm of $\mathbb{A}$. It is clear that the $b_{i j}(m)$ are integers. Since we are assuming $q \geq 5$, $e_{j}=2$ for all $j$ ([P3, Remark 5.26]). The Brandt matrix $B(m)$ is the $H$ by $H$ matrix with $b_{i j}(m)$ as the $i$ th, $j$ th entry.

Proposition 3. Let the notation be as above and assume $m, m^{\prime}$, and $p$ are relatively prime to $N$. Then the ideals $I_{1}, \ldots, I_{H}$ can be ordered so that, simultaneously for all $m$,

$$
B(m)=\left(\begin{array}{cc}
C(m) & 0 \\
0 & C(m)
\end{array}\right) \quad\left(\operatorname{resp} .\left(\begin{array}{cc}
0 & D(m) \\
D(m) & 0
\end{array}\right)\right)
$$

if $m$ is a quadratic residue (resp. nonresidue) $\bmod q$. Further:

(1) $C(m)=\left(c_{i j}(m)\right)$ and $D(m)=\left(d_{i j}(m)\right)$ are $H / 2$ by $H / 2$ symmetric matrices and depend, up to conjugation by a permutation matrix, only on the level $N=q^{2} M$, not on the particular order $\mathscr{O}$, nor on the ideal class representatives used to define them.

(2) If $m$ is a quadratic residue mod $q$ then $\sum_{j=1}^{H / 2} c_{i j}(m)=$ $\sigma_{1}(m)$ for all $i, 1 \leq i \leq H / 2$ while if $m$ is a quadratic nonresidue mod $q$ then $\sum_{j=1}^{H / 2} d_{i j}(m)=\sigma_{1}(m)$ for all $i$, $1 \leq i \leq H / 2$. Here $\sigma_{r}(m)=\sum_{d \mid m} d^{r}$, the sum being over all positive divisors of $m$.

(3) The $B(m)$ form a commuting family of diagonalizable matrices which satisfy the following relations:

$$
\begin{array}{ll}
B(m) B\left(m^{\prime}\right)=B\left(m m^{\prime}\right) & \text { if }\left(m, m^{\prime}\right)=1 \\
B\left(p^{r}\right) B\left(p^{s}\right)=\sum_{k=0}^{\min \{r, s\}} p^{k} B\left(p^{r+s-2 k}\right), & \text { if } p \text { is prime. }
\end{array}
$$


(4) If $m$ is a quadratic residue mod $q$, then $\lambda_{0}=\sigma_{1}(m)$ is an eigenvalue of $C(m)$ and the other eigenvalues $\lambda_{i}, 1 \leq i \leq$ $H / 2-1$ satisfy $\left|\lambda_{i}\right| \leq \sigma_{0}(m) \sqrt{m}$. If $m$ is a quadratic nonresidue $\bmod q$, then $\lambda_{0}=\sigma_{1}(m)$ and $\lambda_{H-1}=-\sigma_{1}(m)$ are eigenvalues of $B(m)$ and the other eigenvalues $\lambda_{i}, \quad 1 \leq i \leq$ $H-2$ satisfy $\left|\lambda_{i}\right| \leq \sigma_{0}(m) \sqrt{m}$.

Sketch of proof. This follows from [P3]. The eigenvalues $\lambda$ of $B(m)$ not equal to $\pm \sigma_{1}(m)$ are eigenvalues for the action of $T_{m}$ on a space of cuspforms of weight 2 on $\Gamma_{0}(N)$ and so $|\lambda| \leq$ $\sigma_{0}(m) \sqrt{m}$ by the Petersson Ramanujan Conjecture (see, e.g., $[\mathrm{K}]$ p. 164 ) which was proved by Deligne ([D]).

Finally we remark that an explicit formula for the trace of the $B(m)$ is given by Theorem 4.12 of [P3]. Trace formulas for related cases can be found in [E, HS, P1], and, for the most general case, in [HPS1].

\section{Ramanujan and Related GRaphS}

Let the notation and assumptions be as in $\S 2$. Assume that all diagonal entries of $B(m)=B\left(q^{2}, M ; m\right)$ are even and let $G(m)=G\left(q^{2}, M ; m\right)$ denote the multigraph whose adjacency matrix is $B(m)$ (resp. $C(m)$ ) if $m$ is a nonresidue (resp. residue) mod $q$. In other words, let $G^{\prime}(m)$ denote the graph whose vertices are identified with the $H$ left ideal classes of $\mathscr{O}$ represented by $I_{1}, \ldots, I_{H} \cdot G^{\prime}(m)$ has an edge connecting $I_{i}$ and $I_{j}$ for each pair $\pm \alpha \in I_{j}^{-1} I_{i}$ with $\mathrm{N}(\alpha)=m \mathrm{~N}\left(I_{i}\right) / \mathrm{N}\left(I_{j}\right) . G(m)=G^{\prime}(m)$ if $m$ is a nonresidue $\bmod q$. If $m$ is a residue $\bmod q, G^{\prime}(m)$ consists of two isomorphic connected components and $G(m)$ denotes one of these components. For a real number $x$, let $\lceil x\rceil$ denote the smallest integer greater than or equal to $x$.

Theorem 1. Let $\mathscr{O}$ be an order of level $N=q^{2} M$ in $\mathbb{A}$ with class number $H$ given by (4) and let $p$ be a prime with $p<q / 4$ and $p \nmid N$. Then the associated multigraph $G(p)=G\left(q^{2}, M ; p\right)$ is defined and is a $p+1$ regular connected Ramanujan graph. Ramanujan means that all eigenvalues $\lambda$ of the adjacency matrix not equal to $\pm(p+1)$ satisfy $|\lambda| \leq 2 \sqrt{p}$ which is asymptotically best possible. $G(p)$ has no even cycles of length $s<2\left\lceil\log _{p} q-\log _{p} 4\right\rceil$ and no odd cycles of length $s<\left\lceil\log _{p} q-\log _{p} 4\right\rceil$. Assume $p$ is a residue (resp. nonresidue) mod $q$. Then $G(p)$ is nonbipartite (resp. bipartite) of order $n=|G(p)|=H / 2$ (resp. $H$ ) with girth $g$ and diameter 
$d$ satisfying $g \geq\left\lceil\log _{p} q-\log _{p} 4\right\rceil$ and $d \leq 2 \log _{p} n+2$ (resp. $g \geq 2\left\lceil\log _{p} q-\log _{p} 4\right\rceil$ and $\left.d \leq 2 \log _{p} n+2 \log _{p} 2+1\right)$.

Sketch of proof. The results, excluding those on cycles and diameter, follow from Proposition 3. The diameter result is a consequence of the Ramanujan property (see Theorem 5.1 of [LPS3]). If $s$ is odd (resp. even) and $4 p^{s}<q$ (resp. $4 p^{\frac{s}{2}}<q$ ), it follows from Proposition 2.5 and Theorem 4.12 of [P3] that $\operatorname{tr}\left(B\left(p^{s}\right)\right)=0$ (resp. $H$ ). Then by Proposition 2 above we see that $\operatorname{tr}\left(A_{s}\right)=0$ for $s$ as above and the results on cycles and girth follow.

Remark 2 . Theorem 1 can be greatly generalized. Let $\mathbb{B}$ denote the quaternion algebra over $\mathbb{Q}$ ramified precisely at the distinct primes $q_{1}, \ldots, q_{e}$ and $\infty$ ( $e$ is odd). Let $Q=\prod_{i=1}^{e} q_{i}^{r_{i}}$ with $r_{i} \geq 1$ and let $M$ be a positive integer prime to $Q$. Let $\mathscr{O}$ be an order in $\mathbb{B}$ such that $\mathscr{O}_{\ell}=\mathscr{O} \otimes \mathbb{Z}_{\ell}$ is a maximal order for all primes $\ell+Q M$ and such that $\mathscr{O}_{\ell}, \ell \mid Q M$, has level $\ell^{r}$, $r=\operatorname{ord}_{\ell}(Q M)$, and contains the full ring of integers in a quadratic (field or $Q_{\ell} \oplus Q_{\ell}$ ) extension of $Q_{\ell}$ embedded in $\mathbb{B}_{\ell}=\mathbb{B} \otimes Q_{\ell}$. Level $\ell^{r}$ means that $\mathscr{O}_{\ell}$ has index $\ell^{r-1}$ (resp. $\ell^{r}$ ) in a maximal order of $\mathbb{B}_{\ell}$ if $\ell \mid Q$ (resp. $\ell \mid M$ ). For $\ell \mid Q$, there is (up to isomorphism) a unique such order of level $\ell$ (the maximal order) and level $\ell^{2}$ (the order $\mathscr{O}_{\ell}$ with $\mathscr{O}$ as in Theorem 1 which corresponds to any ramified extension of $Q_{\ell}$ ). Excluding $\ell=2$, for $r \geq 3$ and odd there are 3 such orders of level $\ell^{r}$ (corresponding to the three quadratic field extensions of $\mathbb{Q}_{\ell}$ ) and for $r \geq 4$ and even there are 2 such orders (corresponding to the ramified extensions of $\mathbb{Q}_{\ell}$ ) (see [HPS1]). For $\ell \mid M$, again excluding $\ell=2$, there is a unique such order of level $\ell$ (corresponding to $\mathbb{Q}_{\ell} \oplus \mathbb{Q}_{\ell}$ or to any ramified extension), 3 such orders of level $\ell^{2}$ (corresponding to $\mathbb{Q}_{\ell} \oplus \mathbb{Q}_{\ell}$, the unramified extension, and either ramified extension of $Q_{\ell}$ ). For $r \geq 3$ and odd there are 3 such orders (corresponding to $\mathbb{Q}_{\ell} \oplus \mathbb{Q}_{\ell}$ and the two ramified extensions) and for $r \geq 4$ and even there are 4 such orders (corresponding to all the extensions of $\mathbb{Q}_{\ell}$ ) (see $\left.[\mathrm{Br}, \mathrm{J}, \mathrm{H} 1, \mathrm{H} 2]\right)$. The orders $\mathscr{O}_{\ell}, \ell \mid M$, where $\mathscr{O}$ is as in Theorem 1 , all correspond to $\mathbb{Q}_{\ell} \oplus \mathbb{Q}_{\ell}$. Since all the above choices are independent of each other and since for every choice there exists a distinct order $\mathscr{O}$, there are many such orders. To each such order and Hecke operator $T_{p}, p \nmid Q M$ (for simplicity assume $p$ is prime), we seek to associate a Ramanujan graph $G$. Let $L$ be the product of the distinct primes $\ell$ such that 
$\operatorname{ord}_{\ell}(Q M) \geq 2$ and $\mathscr{O}_{\ell}$ corresponds to a ramified extension of $Q_{\ell}$. Then the girth $g$ of the associated $G$ satisfies $g \geq\left\lceil\log _{p} L-\log _{p} 4\right\rceil$ $\left(\geq 2\left\lceil\log _{p} L-\log _{p} 4\right\rceil\right.$ if $G$ is bipartite). In particular we should assume that $L \neq 1$ and that $p$ is suitably small so that the girth of $G$ is at least 3 which implies that $G$ has neither loops nor multiple edges ( $L=q$ in Theorem 1). Analogous constructions can be done over any totally real number field and should yield interesting graphs. Let us end this long remark by giving a more precise example. Let $Q=\prod_{i=1}^{e} q_{i}^{2}$ and $M=1$ where all $q_{i} \geq 5$, and let $\mathscr{O}$ be an associated order. Let $p<\frac{1}{4} \sqrt{Q}$ be a prime not dividing $Q$ and let $G$ be the graph associated to $T_{p}$ and $\mathscr{O}$. If $p$ is (resp. is not) a quadratic residue for all $q_{i}$, then $G$ is a connected $p+1$ regular nonbipartite (resp. bipartite) Ramanujan graph of order $\frac{H}{2^{e}}$ (resp. $\left.\frac{H}{2^{e-1}}\right)$ and girth $g$ where $H=\frac{1}{12} \prod_{i=1}^{e}\left(q_{i}^{2}-1\right)$ and $g \geq\left\lceil\frac{1}{2} \log _{p} Q-\log _{p} 4\right\rceil$ ( resp. $\geq 2\left\lceil\frac{1}{2} \log _{p} Q-\log _{p} 4\right\rceil$ ).

Theorem 2. Let $\mathscr{O}, \mathbb{A}$, and $H$ be as in Theorem 1 . Let $m, m_{1}$, $\ldots, m_{r}$ be positive, nonsquare integers relatively prime to $Q M$.

$A$. For $m$ a residue (resp. nonresidue) mod $q$ with $4 m<q$, let $G(m)$ be the multigraph whose adjacency matrix $A_{1}$ is $C(m)$ (resp. $B(m)$ ). Then $G(m)$ is a $\sigma_{1}(m)$ regular connected nonbipartite (resp. bipartite) graph of order $H / 2$ (resp. H). All eigenvalues $\lambda$ of $A_{1}$ not equal to $\pm \sigma_{1}(m)$ satisfy $|\lambda| \leq \sigma_{0}(m) \sqrt{m}$. If $4 m^{3}<q$, then $G(m)$ has no cycles of length 3 .

B. Assume $4 m^{2}<q$ and let $G\left(m^{2}\right)$ be the multigraph whose adjacency matrix $A_{1}$ is $C\left(m^{2}\right)-I$ where $I$ is the $H / 2$ by $H / 2$ identity matrix. Then $G\left(m^{2}\right)$ is a $\left(\sigma_{1}\left(m^{2}\right)-1\right)$ regular connected nonbipartite graph of order $H / 2$. All eigenvalues $\lambda$ of $A_{1}$ not equal to $\sigma_{1}\left(m^{2}\right)-1$ satisfy $|\lambda| \leq \sigma_{0}\left(m^{2}\right) m+1$.

C. Assume $m_{1}<m_{2}<\cdots<m_{r}$ are relatively prime in pairs and that $4 m_{r-1} m_{r}<q$. Let $A_{1}=\sum_{i=1}^{r} C\left(m_{i}\right) \quad\left(\right.$ resp. $\left.=\sum_{i=1}^{r} B\left(m_{i}\right)\right)$ if all (resp. not all) of the $m_{i}$ are residues $\bmod q$. Then $A_{1}$ is the adjacency matrix of a regular connected graph of order $H / 2$ (resp. $H$ ) and degree $\sum_{i=1}^{r} \sigma_{1}\left(m_{i}\right)$. If all the $m_{i}$ are residues (resp. non residues) $\bmod q$, then all eigenvalues $\lambda$ of $A_{1}$ not equal to $\pm \sum_{i=1}^{r} \sigma_{1}\left(m_{i}\right)$ satisfy $|\lambda| \leq \sum_{i=1}^{r} \sigma_{0}\left(m_{i}\right) \sqrt{m_{i}}$.

Sketch of proof. These results follow from Propositions 1, 2, and 3 and the properties of Hecke operators.

We call the graphs $G(m)$ in Theorem 2A almost Ramanujan because the nontrivial eigenvalues of the adjacency matrices of these 
graphs satisfy the Ramanujan conjecture bound $\sigma_{0}(m) \sqrt{m}$. The diameter of the graphs in Theorem 2 is bounded by the following general result of Chung ([C]) in the nonbipartite case and its obvious analogue in the bipartite case.

Proposition 4. Let $G$ be a $k$ regular graph of order $n$ and let $\mu$ denote the maximum of the absolute values of all the eigenvalues of the adjacency matrix $A_{1}$ of $G$ not equal to $\pm k$. Assume $\mu<k$ and that $\pm k$ occur with multiplicity at most one in $A_{1}$. If $G$ is nonbipartite, i.e., $-k$ is not an eigenvalue, (resp. bipartite), then $G$ is connected with diameter $d$ satisfying

$$
\left.d \leq \frac{\log (n-1)}{\log (k / \mu)}+1 \quad \text { (resp. } d \leq \frac{\log \left(\frac{n}{2}-1\right)}{\log (k / \mu)}+2\right) .
$$

Note that a similar (often slightly better) diameter bound for $k$ regular graphs can be obtained by applying the method of proof of Theorem 5.1 of [LPS3] to the general case.

By the footnote on p. 20 of [B], all the graphs we have considered have high vertex connectivity.

\section{EXAMPLES}

In this section we give several examples of graphs which are constructed by our method. Let $d$ and $g$ denote the diameter and girth of a graph and see Bien's article [B] for the definitions of magnifier and expander.

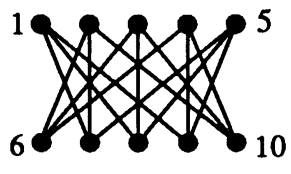

$G\left(11^{2}, 1 ; 2\right)$

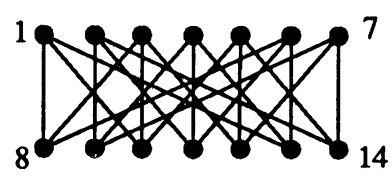

$G\left(13^{2}, 1 ; 2\right)$

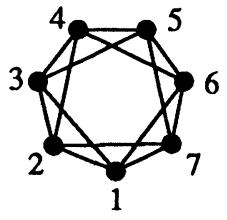

$G\left(13^{2}, 1 ; 3\right)$

$G=G\left(11^{2}, 1 ; 2\right)$ is a $(5,3,5 / 6)$ expander. Theorem 1 gives $d \leq 6.4$ and $g \geq 2\lceil 1.5\rceil$ and in fact $d=3$ and $g=4$. The automorphism group of $G$ has order 48 and is generated by the reflections about the horizontal and vertical axes, the transposition $(1,5)$ and the element $(2,3,4)(7,8,9)$ of order 3 .

$G=G\left(13^{2}, 1 ; 2\right)$ is a $(7,3,7 / 6)$ expander which is best possible. Theorem 1 gives $d \leq 10.7$ and $g \geq 2\lceil 1.7\rceil$ and in fact $d=4$ and $g=4$. The automorphism group of $G$ has order 28 
and is generated by the reflections $R_{H}$ and $R_{V}$ about the horizontal and vertical axes and the translation (or rotation) $T$ given by $(1,2,3,4,5,6,7)(8,9,10,11,12,13,14)$. Note that $T R_{H}$ has order 14 and that the automorphism group is also generated by $T R_{H}$ and $R_{V}$.

$G=G\left(13^{2}, 1 ; 3\right)$ is a $(7,4,4 / 3)$ magnifier which is best possible. Theorem 1 gives $d \leq 5.5$ and $g \geq\lceil 1.07\rceil$ (but Theorem 1 also gives that $G$ is a graph so that $g \geq 3$ ) and in fact $d=2$ and $g=3$. The automorphism group of $G$ has order 14 and is generated by a reflection and a rotation.

As in Theorem $2 \mathrm{C}$ (which doesn't strictly apply in this case) we can consider the graph $G$ whose adjacency matrix is $B\left(13^{2}, 1 ; 2\right)+B\left(13^{2}, 1 ; 3\right)$. This graph is obtained by gluing two copies of $G\left(13^{2}, 1 ; 3\right)$ to $G\left(13^{2}, 1 ; 2\right)$, one to the inputs $v_{1}, \ldots, v_{7}$ and one to the outputs $v_{8}, \ldots, v_{14}$ where, for the outputs, the vertices are identified $\bmod 7$. Then $G$ is a $(14,7,1)$ magnifier which is best possible and has $d=2$ and $g=3$. The automorphism group of $G$ is identical to that of $G\left(13^{2}, 1 ; 2\right)$.

Bien in [B] stated that one is interested in graphs of order about $1,000,000$ and degree about 1,000 . As our final example we determine such a graph. In the process we demonstrate how a particular graph or multigraph can be easily modified to obtain another graph with enhanced properties. Let $q=2003$ and $p=991$ and note that $p$ is a nonresidue $\bmod q$. Thus, if $r$ is odd, $q \nmid\left(s^{2}-4 p^{r}\right)$ for any $s$. It follows from Theorems 4.2 and 2.7 of [P3] that $\operatorname{tr} B\left(q^{2}, 1 ; p^{r}\right)=0$ for $r$ odd. Hence we can associate the multigraph $G^{\prime}=G\left(q^{2}, 1 ; p\right)$ of order $H\left(q^{2}, 1\right)=334,334$ to $B\left(q^{2}, 1 ; p\right)$. Now $q \mid\left(s^{2}-4 p^{2}\right)$ where $s^{2}-4 p^{2}<0$ if and only if $s=21$. It follows that $\operatorname{tr} B\left(q^{2}, 1 ; p^{2}\right)>H\left(q^{2}, 1\right)$ and thus $G^{\prime}$ has girth 2. Now consider level $q^{2} M$ with $M=2$. By the calculations above $\operatorname{tr} B\left(q^{2}, 2 ; p^{r}\right)=0$ for $r$ odd. Also $21^{2}-4 p^{2} \equiv 5 \bmod 8$ so by Theorem 4.2 of [P3] and the tables in [P2] $\operatorname{tr} B\left(q^{2}, 2 ; p^{2}\right)=H\left(q^{2}, 2\right)=1,003,002$. Thus the girth of $G=G\left(q^{2}, 2 ; p\right)$ is at least 4 and a trivial calculation shows it is 4 . Note that (see, e.g., [Bo]) for a bipartite 992 regular graph to have girth greater than 4 , it would have to have order at least $1,966,146$ so the girth of $G$ is best possible for a bipartite graph of order approximately $1,000,000$ and degree approximately 1,000 . We have been able to determine certain information about $G$ by easy hand calculations. However, to explicitly construct $G$, say 
by the algorithm presented in [P4], would require a large amount computing power.

\section{REFERENCES}

[B] F. Bien, Constructions of telephone networks by group representations, Notices Amer. Math. Soc. 36 (1989), 5-22.

[Bo] B. Bollobas, Graph theory, Springer-Verlag, New York, 1979.

[Br] J. Brzezinski, On automorphisms of quaternion orders, 1989, preprint.

[C] F. R. K. Chung, Diameters and eigenvalues, J. Amer. Math. Soc. 2 (1989), 187-196.

[D] P. Deligne, La conjecture de weil I, Publ. Math. I.H.E.S. 43 (1974), 273308.

[E] M. Eichler, The Basis Problem for modular forms and the traces of the Hecke operators, Lecture Notes in Math. No. 320, Springer, Berlin, 1973.

[H1] H. Hijikata, Explicit formula of the traces of the Hecke operators for $\Gamma_{0}(N)$, J. Math. Soc. Japan 26 (1974), 56-82.

[H2] - Private communication, 1988.

[HS] H. Hijikata, and H. Saito, On the representability of modular forms by theta series, No. Theory, Alg. Geo., and Comm. Alg. (in honor of Y. Akizuki), Kinokuniya, Tokyo, pp. 13-21.

[HPS1] H. Hijikata, A. Pizer, and T. Shemanske, Orders in quaternion algebras, J. Reine Angew. Math. 394 (1989), 59-106.

[HPS2] H. Hijikata, A. Pizer, and T. Shemanske, The Basis Problem for Modular Forms on $\Gamma_{0}(N)$, Mem. Amer. Math. Soc. 82, No. 418 (1989).

[I] Y. Ihara, Y., Discrete subgroups of PL $\left(2, k_{p}\right)$, Proc. Sympos. in Pure Math., vol. IX, 1966, pp. 272-278.

[J] S. Jun, Private communication, 1988.

[K] N. Koblitz, Introduction to elliptic curves and modular forms, SpringerVerlag, New York, 1984.

[L] W. Li, Abelian Ramanujan Graphs, 1989, preprint.

[LPS1] A. Lubotzky, R. Phillips, and P. Sarnak, Explicit expanders and the Ramanujan conjectures, Proc. of the Eighteenth Annual ACM Sympos. on Theory of Computing 18 (1986), 240-246.

[LPS2] A. Lubotzky, R. Phillips, and P. Sarnak, Hecke operators and distributing points on $S^{2}$. II, Comm. Pure Appl. Math. XL (1987), 401-420.

[LPS3] A. Lubotzky, R. Phillips, and P. Sarnak, Ramanujan graphs, Combinatorica 8 (1988), 261-277.

[M] G. Margulis, Manuscript in Russian on graphs with large girth, 1987.

[P1] A. Pizer, On the arithmetic of quaternion algebras II, J. Math. Soc. Japan 28 (1976), 676-688.

[P2] A. Pizer, The representability of modular forms by theta series, J. Math. Soc. Japan 28 (1976), 689-698.

[P3] A. Pizer, Theta series and modular forms of level $p^{2} M$, Compositio Math. 40 (1980), 177-241. 
[P4] A. Pizer, An algorithm for computing modular forms on $\Gamma_{0}(N)$, J. of Algebra 64 (1980), 340-390.

[S] J. P. Serre, Trees, Springer-Verlag, New York, 1980.

Department of Mathematics, University of Rochester, Rochester, NEW YORK 14627 
